\title{
Fully Nonlinear Stochastic Partial Differential Equations: Non-Smooth Equations and Applications
}

\section{Equations aux dérivées partielles stochastiques complètement non linéaires : équations non régulières et applications}

\author{
Pierre Louis Lions \\ CEREMADE-UMR 7534 \\ Université de Paris-Dauphine \\ Place de Lattre de Tassigny \\ 75775 Paris Cedex 16 \\ FRANCE
}

\author{
Panagiotis E. Souganidis \\ Department of Mathematics \\ University of Wisconsin-Madison \\ Madison, WI 53706 \\ USA
}

and

\begin{abstract}
In this note, we extend the result described in a previous note to the case of non-smooth Hamiltonians for fully nonlinear stochastic partial differential equations. And we present some applications of our theory to pathwise stochastic control and to the propagation of fronts in random environments.
\end{abstract}

Résumé : Dans cette note, nous étendons les résultats décrits dans une note précédente au cas d'Hamiltoniens non réguliers pour des équations aux dérivées partielles stochastiques complètement nonlinéaires. Et nous présentons quelques applications de notre théorie au contrôle stochastique trajectoriel et à la propagation de fronts dans des environnements aléatoires.

\section{Version Française Abrégée :}

Nous étendons ici les résultats obtenus dans [LS1] à des équations plus générales (pour des Hamiltoniens peu réguliers) et présentons quelques applications de cette théorie. Plus précisément, nous considérons des équations paraboliques sltochastiques, éventuellement dégénérées, du second ordre, complètement nonlinéaires qui s'écrivent 
sous la forme

$$
\begin{cases}d u=F\left(D^{2} u, D u\right) d t+\sum_{i=1}^{M} H_{i}(D u) \circ d W_{i} & \text { in } \mathbb{R}^{N} \times(0, \infty), \\ u=u_{0} & \text { on } \mathbb{R}^{N} \times\{0\} .\end{cases}
$$

où $u_{0}$ est bornée, uniformément continue sur $\mathbb{R}^{N}, W$ est un Brownien standard dans $\mathbb{R}^{M}$, ○ correspond à la différentielle de Stratonovich, et $F$ est continue sur $\mathbb{R}^{N} \times S^{N}$ (l'espace des matrices symétriques $N \times N)$ et elliptique dégénérée, i.e.

$$
F(X, p) \leq F(Y, p), \quad \forall p \in R^{N}, \quad \forall X \leq Y \in \mathcal{S}^{N}
$$

La principale extension, par rapport aux résultats de [LS1], est la prise en compte d'Hamiltoniens $H_{1}, \ldots, H_{M}$ non nécessairement très réguliers ( $C^{3}$ dans [LS1]) puisque nous supposons que $H_{i}$ est Lipschitzien sur $\mathbb{R}^{N}$ et peut s'écrire comme la différence de deux fonctions convexes. Cette extension est nécessaire pour pouvoir appliquer notre théorie à la propagation de fronts dans des milieux aléatoires, où typiquement $M=1$, et $H(D u)=|D u|$.

Dans la première partie de cette note, nous montrons comment il est possible d'étendre et adapter les résultats de [LS1] et en particulier la convergence des solutions approchées, obtenus par régularisation du mouvement Brownien $W$, vers une limite unique indépendante de l'approximation choisie.

Nous présentons ensuite quelques applications de la théorie développée dans [LS1] et ici, et notamment i) au contrôle stochastique trajectoriel, ii) à la propagation de fronts dans des milieux aléatoires, et iii) à des problèmes asymptotiques pour les questions de transition de phase dans les environnements stochastiques.

\section{INTRODUCTION}

In this note we extend the theory of parabolic, possibly degenerate, second-order, stochastic partial differential equations we introduced in [LS1] to equations with less regularity and present several applications. In particular we consider equations, which 
written in the Stratonovich sense have the form

$$
\begin{cases}d u=F\left(D^{2} u, D u\right) d t+\sum_{i=1}^{M} H_{i}(D u) \circ d W_{i} & \text { in } \mathbb{R}^{N} \times(0, \infty), \\ u=u_{0} & \text { on } \mathbb{R}^{N} \times\{0\} .\end{cases}
$$

As in $[\mathrm{LS} 1], u_{0} \in B U C\left(\mathbb{R}^{N}\right)$, the space of bounded uniformly continuous on $\mathbb{R}^{N}$, $W=\left(W_{1}, \ldots, W_{M}\right)$ is the standard $M$-dimensional Brownian motion in time and, hence, $d W=\left(d W_{1}, \ldots, d W_{M}\right)$ is the "usual" $M$-dimensional White noise in time and $F$ is continuous and degenerate elliptic, i.e., it satisfies, for all $X, Y \in \mathcal{S}^{N}$, the space of $N \times N$ symmetric matrices, and $p \in \mathbb{R}^{N}$,

$$
\text { if } X \leq Y, \text { then } F(X, p) \leq F(Y, p) \text {. }
$$

The main difference with [LS1] is that, instead of $H \in C^{3}\left(\mathbb{R}^{N} ; \mathbb{R}^{M}\right)$, here we only assume that

$$
\left\{\begin{array}{l}
H \in C^{0,1}\left(\mathbb{R}^{N} ; \mathbb{R}^{M}\right) \text { and, for each } i \in\{1, \ldots, M\} \\
H_{i} \text { can be written as the difference of two convex functions. }
\end{array}\right.
$$

The second part of assumption (0.3) is a technical one; in Section 1 below we point out where it is used. Notice, however, that any $H \in C^{0,1}\left(\mathbb{R}^{N} ; \mathbb{R}^{M}\right)$ which is positively homogeneous of degree 1, an important class of $H$ 's for the applications we present below, satisfies (0.3).

Equations like (0.1) appear in a variety of applications like asymptotics of equations with rapidly oscillating (mixing) coefficients in time, front propagation and phase transitions in random media, pathwise stochastic control theory, Zakai equations in filtering and stochastic control with partial observations, mathematical finance, etc.. In this note we outline some applications to pathwise stochastic control and to front propagation and phase transitions in random media.

Our theory extends to $(x, t, \omega)$-dependent equations with $d W(t, x)$ an $M$-dimensional white noise, which is regular is $x$. We refer to [LS1] for a discussion of this as well as other possible extensions and to [LS2,3] for the details. 
In spite of their importance very little was known for equations like (0.1) even for smooth $H$ before [LS1], with the exception of the uniformly elliptic linear theory, i.e., when both $F$ and $H$ are linear and $F$ is uniformly elliptic — see, for example, Watanabe [W] and the references therein — and some uniformly elliptic quasilinear cases — see Pardoux $[\mathrm{P}]$.

The main difficulty about equations like $(0.1)$ is the well-known fact, even in the deterministic case, that there are no global smooth solutions in general. Moreover, the fully nonlinear character of the equations seems to make them inaccessible to the classical martingale theory employed for the linear case. Finally, even when smooth solutions may exist, the equations can not be described in a pointwise sense, because of the everywhere lack of differentiability of the Brownian motion. In the deterministic case the lack of regularity was overcome with the introduction by Crandall and Lions [CL] of the notion of viscosity solutions - we refer to [CIL], [B], [FS] and [BCESS] for an up-to-last year overview of the theory of viscosity solutions and their applications in the deterministic setting.

For $H \in C^{3}\left(\mathbb{R}^{N} ; \mathbb{R}^{M}\right)$, the aforementioned difficulties were overcome in [LS1] by proving that all possible deterministic approximations of (0.1) converge uniformly in $(x, t) \in \mathbb{R}^{N} \times[0, T]$, for all $T>0$, and a.s. in $\omega$ to the same limit, which is then interpreted as a stochastic viscosity solution of (0.1), which, in some cases, was proved to be unique in its class. Here we extend [LS1] to the case that (0.3) holds. We present these results in Section 1, while in Section 2 we present some applications of the theory.

\section{THE EXISTENCE AND NOTION OF WEAK SOLUTIONS}

We consider the following class of approximations to $(0.1)$

$$
\begin{cases}u_{t}^{\varepsilon}=F\left(D^{2} u^{\varepsilon}, D u^{\varepsilon}\right)+\sum_{i=1}^{M} H_{i}\left(D u^{\varepsilon}\right) \dot{\zeta}_{i}^{\varepsilon}(t) & \text { in } \mathbb{R}^{N} \times(0, \infty), \\ u^{\varepsilon}=u_{0}^{\varepsilon} & \text { on } \mathbb{R}^{N} \times\{0\},\end{cases}
$$

where $u_{0}^{\varepsilon}, u_{0} \in B U C\left(\mathbb{R}^{N}\right)$ and the smooth functions $\zeta^{\varepsilon}=\left(\zeta_{1}^{\varepsilon}, \ldots, \zeta_{M}^{\varepsilon}\right):[0, \infty] \times \Omega \rightarrow$ $\mathbb{R}^{M}$ are such that, as $\varepsilon \rightarrow 0$ and for all $T>0$,

$$
\zeta^{\varepsilon} \rightarrow W \text { uniformly in }[0, T] \text { and a.s. and } u_{0}^{\varepsilon} \rightarrow u_{0} \text {. }
$$


To obtain the convergence of $\left(u^{\varepsilon}\right)_{\varepsilon>0}$ we need the following technical assumption on $F$. We refer to [LS1] and the references therein for a discussion of (1.3) and the existence of $\zeta^{\varepsilon}$ as in (1.2).

$$
\left\{\begin{array}{l}
\text { There exists } G \in C\left(S^{2 N} \times \mathbb{R}^{N}\right) \text { degenerate elliptic such that, for } \\
\text { (i) } G\left(\begin{array}{cc}
\lambda X & -\lambda X \\
-\lambda X & \lambda X
\end{array}, p\right)=0 \text {, for all } \lambda \in \mathbb{R} \text { and all } X \in \mathcal{S}^{N} \\
\text { and } \\
\text { (ii) } F(X, p)-F(Y, p) \leq G(Z, p), \\
\quad \text { for all } X, Y \in \mathcal{S}^{N} \text { and } Z \in \mathcal{S}^{2 N} \text { such that }\left(\begin{array}{cc}
X & 0 \\
0 & Y
\end{array}\right) \leq Z
\end{array}\right.
$$

The result is:

Theorem 1.1. Let $\left(\zeta^{\varepsilon}\right)_{\varepsilon>0}$ and $\left(\xi^{\eta}\right)_{\eta>0}$ satisfy (1.2). Assume that $F$ satisfies (0.2) and (1.3) and that $H$ satisfies (0.3). If $\left\|u_{0}^{\varepsilon}-v_{0}^{\eta}\right\| \rightarrow 0$, as $\varepsilon, \eta \rightarrow 0$, then $\lim _{\varepsilon, \eta \rightarrow 0}\left\|u^{\varepsilon}-v^{\eta}\right\|_{C\left(\mathbb{R}^{N} \times[0, T]\right)}=0$, for every $T>0$ and a.s. in $\omega$, where $u^{\varepsilon}$ and $v^{\eta}$ solve (1.1) with initial data $u_{0}^{\varepsilon}$ and $v_{0}^{\eta}$ respectively. In particular, each family $\left(u^{\varepsilon}\right)_{\varepsilon>0}$ is Cauchy in $\mathbb{R}^{N} \times[0, T]$ a.s. in $\omega$, and hence, it converges, uniformly in $(x, t)$ and a.s. in $\omega$, to a unique $u \in B U C\left(\mathbb{R}^{N} \times[0, T]\right)$.

Sketch of the Proof: 1. The theorem follows if we can establish that, a.s. in $\omega$,

$$
\lim _{\lambda \rightarrow 0} \sup _{(x, y, t) \in \mathbb{R}^{2 N} \times[0, T]} \varlimsup_{\varepsilon, \eta \rightarrow 0}\left(u^{\varepsilon}(x, t)-v^{\eta}(y, t)-\lambda|x-y|^{2}\right)=0 .
$$

2. Fix $\lambda>0$, let $\phi(x, y)=\lambda|x-y|^{2}$ and denote by $S^{\varepsilon, \eta}(s, t) \phi(x, y)$ the viscosity solution of

$$
\begin{cases}v_{s}=\sum_{i=1}^{M} H_{i}\left(D_{x} v\right) \dot{\zeta}_{i}^{\varepsilon}-H_{i}\left(-D_{y} v\right) \dot{\xi}_{i}^{\eta} & \text { in } \mathbb{R}^{N} \times \mathbb{R}^{N} \times[t, \infty), \\ v(\cdot, t)=\phi & \text { on } \mathbb{R}^{N} \times \mathbb{R}^{N}\end{cases}
$$

Assumption (0.3) allows us to use Hopf's formula for the unique viscosity solution of (1.5) to prove that, as $\varepsilon, \eta \rightarrow 0$, a.s. in $\omega$ and uniformly in $x, y, s$ and $t$, for $(s, t)$ bounded,

$$
S^{\varepsilon, \eta}(t, s) \phi(x, y) \rightarrow \lambda|x-y|^{2} .
$$


3. The classical theory of viscosity solutions (see [CIL]) yields, in view of (1.3), that if, $z^{\varepsilon, \eta}(x, y, t, s)=u^{\varepsilon}(x, t)-v^{\eta}(y, t)-\mathcal{S}^{\varepsilon, \eta}(t, s) \phi(x, y)$, then

$$
Z_{t}^{\varepsilon, \eta} \leq 0
$$

4. Now (1.6) and (1.7) yield (1.4) arguing by contradiction.

In [LS1] we put forward an intrinsic definition for a weak solution of (0.1) which was based on the existence, for smooth $\phi$, of local time smooth solutions of the stochastic Hamilton-Jacobi equation

$$
\begin{cases}d v=\sum_{i=1}^{M} H_{i}(D v) \circ d W_{i} & \text { in } \mathbb{R}^{N} \times[t, \infty), \\ v=\phi & \text { on } \mathbb{R}^{N} \times\{t\}\end{cases}
$$

given by the method of characteristics, provided $H$ is smooth, for example $C^{3}$. This is not, however, possible in the case at hand. On the other hand, Theorem 1.1 identifies, for any $\phi$, an a.s. unique function, which can be thought as a global solution of (1.8). To state our definition for a weak solution of (0.1), we consider functions (for any $\lambda \in \mathbb{R}$, $p \in \mathbb{R}^{N}$ and $\left.x_{0} \in \mathbb{R}^{N}\right) \phi(x)=\lambda\left|x-x_{0}\right|^{2}+p\left(x-x_{0}\right)$ and denote by $\phi(x, s ; t)$ the function obtained by Theorem 1.1 as it applies to (1.8). We have:

Definition 1.1. A function $u: \mathbb{R}^{N} \times[0, T] \times \Omega \rightarrow \mathbb{R}$ is a subsolution (resp. supersolution) of (0.1) for $F \equiv 0$, if $u(\cdot, \cdot, \omega) \in B U C\left(\mathbb{R}^{N} \times[0, T]\right)$ a.s., and, for all $t \in[0, T),\left(\right.$ resp. $\inf _{x \in \mathbb{R}^{N}}(u(x, s)-\phi(x, s ; t))$ is nonincreasing (resp. nondecreasing)) $s \mapsto \sup _{x \in \mathbb{R}^{N}}(u(x, s)-\phi(x, s ; t))$

Using Theorem 1.1 and a Trotter-Kato formula-type argument as in the proof of Theorem 2.3 of [LS1] we can prove the following:

Theorem 1.2. Assume that $F \equiv 0$ and $H$ satisfies (0.3). Then for each $u_{0} \in B U C\left(\mathbb{R}^{N}\right)$, (0.1) admits a unique weak solution.

We conclude this section remarking that stability results analogous to the one's stated in [LS1] hold here too. 


\section{APPLICATIONS}

(i) Pathwise stochastic control theory.

In a typical stochastic control problem one is given a stochastic differential equation

$$
\left\{\begin{array}{l}
d X_{t}=b\left(x_{t}, \alpha_{t}\right) d t+\widetilde{\sigma}\left(X_{t}, \alpha_{t}\right) \circ d \widetilde{W}_{t}+\sigma\left(X_{t}\right) \circ d W_{t}, \quad(t \geq 0) \\
X_{0}=x
\end{array}\right.
$$

where $\widetilde{W}$ and $W$ are two independent Brownian motions and $\alpha . \in \mathcal{A}$ is a control process, and a pay-off functional

$$
J\left(x, t, \alpha_{.}\right)=g\left(X_{t}\right)+\int_{0}^{t} f\left(X_{s}, \alpha_{s}\right) d s .
$$

In the conventional stochastic control theory the goal is to minimize the mean of $J$, i.e., $\widetilde{\widetilde{\mathbb{E}}} J(x, t, \alpha$. $)$, where $\widetilde{\widetilde{\mathbb{E}}}$ denote the expectation with respect to both Brownian motions, over all possible controls. This leads to the classical Belman equation.

Our theory of stochastic pde allows for trying to minimize $J$ over all controls pathwise, i.e. in an a.s. sense. Moreover, it allows the coefficients to be themselves random. More precisely, if $\widetilde{\mathbb{E}}$ is the expectation with respect to $\widetilde{W}$, define

$$
u(x, t, \omega)=\inf _{\alpha \in \mathcal{A}} \widetilde{\mathbb{E}} J(x, t, \alpha ., \omega)
$$

The following holds:

Theorem 2.1. The function $u$ is a weak solution of

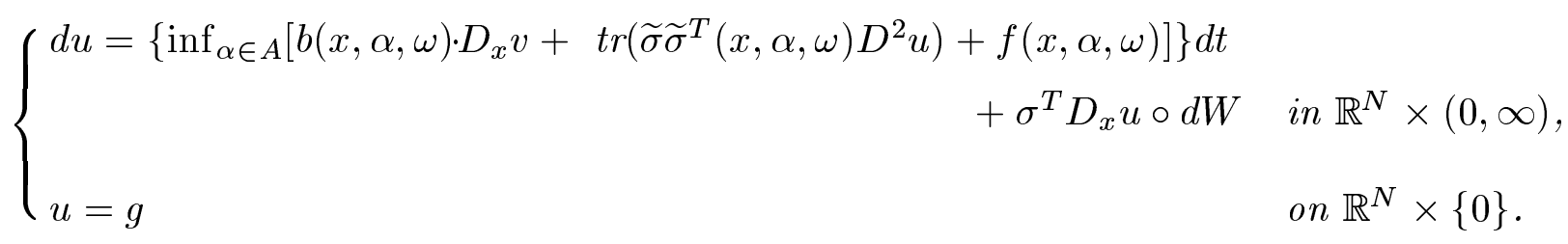

Moreover, $u$ is the a.s. unique limit of the values of the stochastic control problems obtained by approximating $W$.

Since in the above equation $H(p)=\sigma^{T} p \in C^{3}\left(\mathbb{R}^{N} ; \mathbb{R}\right)$, we use the notion of weak solution introduced in [LS1]. 
(ii) Front propagation in random environments.

The interest here is in describing the evolution of (open) sets with normal velocity

$$
V=v_{1}(D n, n) d t+v_{2}(n) \circ d W
$$

where $n$ denotes the normal to the set, past the first time that singularities occur. When $v_{2} \equiv 0$, this goal has been accomplished using the so-called level set approach and its equivalent reformulations. We refer to [BCESS] and [BS] for a general overview of the theory and its numerous application.

Our theory of stochastic pde like (0.1) with $F$ and $H$ satisfying $(0.2)$ and $(0.3)$ allows for the study of evolutions given by (2.1). The level-set pde in this case has the form

$$
d u=F\left(D^{2} u, D u\right) d t+H(D u) \circ d W
$$

with $F$ and $H$ satisfying

$$
\left\{\begin{array}{c}
F \in C\left(S^{N} \times \mathbb{R}^{N} \backslash\{0\}\right) \text { is degenerate elliptic and geometric, } \\
\text { i.e., for all }(X, p) \in S^{N} \times \mathbb{R}^{N} \backslash\{0\}, \lambda>0 \text { and } \mu \in \mathbb{R} \\
F(\lambda X+\mu(p \otimes p), \lambda p)=\lambda F(X, p)
\end{array}\right.
$$

and

$$
H \in C^{0,1}\left(\mathbb{R}^{N}\right) \text { is positively homeogeneous of degree one. }
$$

Consider next the geometric pde of the form

$$
\begin{cases}u_{t}^{\varepsilon}=F\left(D^{2} u^{\varepsilon}, D u^{\varepsilon}\right)+H\left(D u^{\varepsilon}\right) \dot{\zeta}^{\varepsilon} & \text { in } \mathbb{R}^{N} \times(0, \infty), \\ u^{\varepsilon}=u_{0}^{\varepsilon}, & \text { on } \mathbb{R}^{N} \times\{0\},\end{cases}
$$

where $\zeta^{\varepsilon}$ is any approximation of the Brownian motion. We can now define, using Theorem 1.1, an a.s. unique function $u \in B U C\left(\mathbb{R}^{N} \times[0, T] \times \Omega\right)$ obtained as the uniform in $(x, t)$, for $t$ bounded, limit of the $u^{\varepsilon}$ 's, whose level sets are defined to move with normal velocity given by (2.1). Approximations to (2.3) corresponding to the mean curvature 
operator and $H(p)=|p|$ and for convex initial sets were studied using different methods by Yip $[\mathrm{Y}]$.

(iii) Asymptotic problems in phase transitions

We present here an example of an asymptotic problem arising in phase transitions — see [BCESS] and [BS] for an extended discussion of such problems in the deterministic setting and [LS2,3] for more general problems in random environments. The problem is about a modified Allen-Cahn equation of the form

$$
\begin{cases}u_{t}^{\varepsilon}-\Delta u^{\varepsilon}+\varepsilon^{-2}\left(f^{\prime}\left(u_{\varepsilon}\right)+\varepsilon \dot{\zeta}_{\varepsilon}(t)\right)=0 & \text { in } \mathbb{R}^{N} \times[0, T], \\ u^{\varepsilon}=u_{0} & \text { on } \mathbb{R}^{N} \times\{0\},\end{cases}
$$

where $f$ is a double-well potential with wells at \pm 1 of equal depth and $\zeta_{\varepsilon}$ is a smooth function of time. The asymptotics of (2.6), as $\varepsilon \rightarrow 0$, when $\dot{\zeta}_{\varepsilon}(t) \rightarrow c(t)$, with $c(t)$ continuous, were studied in [BSS]. It was shown that, as $\varepsilon \rightarrow 0, u^{\varepsilon} \rightarrow+1$, (resp. -1) locally uniformly inside (resp. outside) a front moving with normal velocity $V=$ mean curvature $+\alpha c(t)$, for some $\alpha$ depending on $f$. When $\dot{\zeta}^{\varepsilon} \rightarrow d W$, this problem was introduced by Funaki [F], who studied its asymptotics for $N=2$ and only for convex sets.

To state our result here we assume that

$$
\partial\left\{u_{0}>0\right\}=\partial\left\{u_{0}<0\right\}=\left\{u_{0}=0\right\}
$$

and, for any approximation $\zeta^{\varepsilon}$ of the Brownian motion, we consider the solution $U^{\varepsilon} \in B U C\left(\mathbb{R}^{N} \times(0, \infty)\right)$ of

$$
\begin{cases}U_{t}^{\varepsilon}-\operatorname{tr}\left[\left(I-\left(\widehat{D U}^{\varepsilon} \otimes \widehat{D U}^{\varepsilon}\right)\right) D^{2} U^{\varepsilon}\right]-\alpha\left|D U^{\varepsilon}\right| \dot{\zeta}^{\varepsilon} & \text { in } \mathbb{R}^{n} \times(0, \infty) \\ U^{\varepsilon}=\mathbf{1}_{\left\{u_{0} \geq 0\right\}}-\mathbf{1}_{\left\{u_{0}<0\right\}} & \text { on } \mathbb{R}^{N} \times\{0\}\end{cases}
$$

where for $p \in \mathbb{R}^{M} \backslash\{0\}, \hat{p}=|p|^{-1} p$, and $\mathbf{1}_{A}$ denotes the characteristic function of the set.

Our theory yields the following result. 
Theorem 2.2. Assume (2.7) and $f^{\prime}( \pm 1)>0$. There exists a constant $\alpha$ such that, as $\varepsilon \rightarrow 0, u^{\varepsilon} \rightarrow+1$ (resp. - 1) locally uniformly in $(x, t)$ and a.s. in $\omega$ inside (resp. outside) a front moving with normal velocity $V=$ mean curvature $+\alpha d W$, i.e., $u^{\varepsilon} \rightarrow 1$ in $\{U>0\}$ and $u^{\varepsilon} \rightarrow-1$ is $\{U<0\}$, where $U$ is the a.s. unique limit of the solutions $U^{\varepsilon}$ of (2.8).

Acknowledgements: This work was partially supported by the TMR network "Viscosity Solutions and their Applications". The second author was support by NSF and ONR. 


\section{REFERENCES}

[B] G. Barles, Solutions de viscosité des équations de Hamilton-Jacobi, Mathématiques et Applications, 17, Springer Verlag, 1994.

[BCESS] M. Bardi, M. G. Crandall, L. C. Evans, H. M. Soner and P. E. Souganidis, Viscosity solutions and applications, Lecture Notes in Mathematics, 1660, Springer Verlag, 1997.

[BS] G. Barles and P. E. Souganidis, A new approach to front propagation: Theory and Applications, Arch. Rat. Mech. Anal.

[BSS] G. Barles, H. M. Soner and P. E. Souganidis, Front propagation and phase field theory, SIAM J. Cont. Opt. 31 (1993), 439-469.

[CIL] M. G. Crandall, H. Ishii and P.-L. Lions, User's guide to viscosity solutions of second order partial differential equations, Bull. AMS 27 (1992), 1-67.

[CL] M. G. Crandall and P.-Lions, Viscosity solutions of Hamilton-Jacobi equations, Trans. AMS 277 (1983), 1-42.

[F] T. Funaki, Singular limit for stochastic reaction-diffusion equation and generation of random interfaces, preprint.

[FS] W. H. Fleming and H. Soner, Controlled Markov processes and viscosity solutions, Springer-Verlag, 1993.

[LS1] P.-L. Lions and P. E. Souganidis, Fully nonlinear stochastic partial differential equations: non smooth equations and applications, CRAS, to appear.

[LS2] P.-L. Lions and P. E. Souganidis, Fully nonlinear stochastic partial differential equations I: the $x$-independent theory, in preparation.

[LS3] P.-L. Lions and P. E. Souganidis, Fully nonlinear stochastic partial differential equations II: the $x$-independent theory, in preparation.

[P] E. Pardoux, Equations aux dérivées partielles stochastiques non linéaires monotones. Etude de solutions fortes de type Ito. These Université Paris Nord, November 1975 . 
[W] H. Watanabe, On the convergence of partial differential equations of parabolic type with rapidly oscillating coefficients of stochastic partial differential equations, Applied Math. Optim. 20 (1989) 81-96.

[Y] N. K. Yip, preprint. 